\title{
Effects of Walking Speed and Step Frequency on Estimation of Physical Activity Using Accelerometers
}

\author{
Jonghoon Park ${ }^{1)}$, Kazuko Ishikawa-Takata ${ }^{1)}$, Shigeho Tanaka ${ }^{1)}$, Yuko Mekata ${ }^{1)}$ and Izumi Tabata ${ }^{1), 2)}$ \\ 1) Health Promotion and Exercise Program, National Institute of Health and Nutrition \\ 2) Faculty of Sport and Health Science, Ritsumeikan University
}

\begin{abstract}
This study evaluated the accuracy of assessing step counts and energy costs under walking conditions altered by step frequency changes at given speeds using uni- (LC) and tri-axial accelerometers (AM, ASP). Healthy young men and women $(n=18)$ volunteered as subjects. Nine tests were designed to manipulate three step frequencies, low $(-15 \%$ of normal), normal, and high $(+15 \%)$, at each walking speed (55, 75 , and $95 \mathrm{~m} / \mathrm{min}$ ). A facemask connected to a Douglas bag was attached to subjects, who wore accelerometers around their waist. LC underestimated the step counts at normal or high step frequency at $55 \mathrm{~m} / \mathrm{min}$ and AM also at all step frequencies at $55 \mathrm{~m} / \mathrm{min}$, whereas ASP did not in all trials. LC underestimated metabolic equivalents (METs) at low or normal step frequency at all walking speeds. AM underestimated METs at low step frequency at all walking speeds and at high step frequency of $95 \mathrm{~m} / \mathrm{min}$. ASP gave underestimates only at low step frequency of $95 \mathrm{~m} / \mathrm{min}$. The degree of the percentage error of METs for AM and ASP was affected by step frequency. Significant interaction between step frequency and speed was found that for LC. These results suggest that $\mathrm{LC}$ and AM can cause errors in step-count functions at a low walking speed. Furthermore, LC may show low accuracy of the METs measurement during walking altered according to step frequency and speed, whereas AM and ASP, which are tri-axial accelerometers, are more accurate but the degree of the percentage error is affected by step frequency. $J$ Physiol Anthropol 30(3): 119-127, 2011 http://www.jstage.jst.go.jp/ browse/jpa2
\end{abstract}

[DOI: 10.2114/jpa2.30.119]

Keywords: accelerometers, step frequency, step counts, energy costs

\section{Introduction}

Walking is the most basic form of human locomotion and one of the most important components of many daily physical activities. An increased amount of energy expended in daily walking reportedly has significant effects on suppressing the progress of various diseases (Hakim et al., 1999; Lee and Buchner, 2008). Thus, walking represents a significant index of human health. Generally, human walking is complexly composed of the multidirectional characteristics of movements altered by combinations of speed and step frequency. Therefore, it would be of great value to accurately assess the number of steps or energy costs expended by various walking movements.

Pedometers are the simplest and the most inexpensive method that has been used for objective assessment of physical behaviors (Crouter et al., 2003; Schneider et al., 2004). Pedometers, especially, have the great advantage of providing immediate information on accumulated step counts as a realtime feedback tool to be more physically active (Bravata et al., 2007). Pedometers have been popularly used for normal subjects and certain epidemiological studies in Japan (Hatano 1993; Mitsui et al., 2008) and mechanisms have gradually been developed for detecting a step from using a spring-suspended lever arm (metal-on-metal contact) to using a magnetic reed proximity switch or accelerations (accelerometer). On the other hand, accelerometers are increasingly used to allow researchers to assess energy costs, and they are considered superior to other methods of measuring various physical activity categories, e.g., low or moderate-intensity activities like walking spread throughout the day (Plasqui and Westerterp, 2007; Bassett et al., 2008). Given the importance of quantifying step counts, most accelerometers made in Japan provide information not only on energy costs but also step counts. The Kenz Lifecorder EX (LC; Suzuken Co., Ltd., Nagoya, Japan), a uniaxial accelerometer using vertical acceleration, is already popular in many countries due to its reasonable cost and reliability for measuring the energy cost of walking or daily physical activity (Kumahara et al., 2004; McClain et al., 2007a, b). More recently, the Actimarker (AM; Panasonic Electronic Works Co., Ltd., Osaka, Japan) and the Active Style Pro (ASP; Omron Health Care Co., Ltd., Kyoto, Japan), newer triaxial accelerometers, have just become commercially available. Tri-axial accelerometers for measuring 
daily physical activity are considered more accurate than uniaccelerometers (Plasqui and Westerterp, 2007; Yamada et al., 2009).

Many investigators have assessed the accuracy and reliability of pedometers and accelerometers, respectively, under various walking conditions (Crouter et al., 2003; Schneider et al., 2004). Pedometers or accelerometers are found to be less accurate in capturing step counts precisely at slow speeds below around $55 \mathrm{~m} \cdot \mathrm{min}^{-1}$ (Crouter et al., 2003; Le Masurier and Tudor-Locke, 2003; Le Masurier et al., 2004). Pedometers or accelerometers have their own vertical acceleration 'threshold' to capture a step. So far, low walking speed is the only factor known to lower accuracy in measuring steps; however, it remains unknown whether or not step frequency changes at a fixed walking speed (especially a slower walking speed) would affect accuracy in measuring step counts.

With respect to measuring energy costs, the uni- (vertical) or tri-axial (vertical, anteroposterior, and mediolateral) accelerometers have clearly demonstrated that output has a strong linear relationship with increasing walking speed (Bouten et al., 1994; Kumahara et al., 2004; Rowlands et al., 2007). However, in these studies, step frequency increased concurrently with walking speed. The rate of body movement changed by step length (step frequency) is likely to be proportional to the displacement in the anteroposterior direction (Dillman, 1975). In this case, a triaxial accelerometer using the integral of three accelerations (vertical, anteroposterior, and mediolateral) would be an appropriate method to quantify the energy cost from the multidirectional characteristics of human movement during walking. However, it remains unclear how much step frequency changes at a fixed walking speed would independently affect the accuracy of measuring energy costs with any accelerometer.

Accordingly, the purpose of the present study was to evaluate the accuracy of measuring step-count functions and energy costs from these three accelerometers under walking conditions altered by step frequency changes at given speeds.

\section{Methods}

\section{Subjects}

We recruited 18 healthy adult participants $(9$ male and 9 female, 23-41 yr) who work at the same worksites and did not have any physical impairment that affected ambulation. The descriptive characteristics of the subjects are presented in Table 1. All aspects of this study were approved by the Ethical Committee of the National Institute of Health and Nutrition. All subjects signed an informed consent document before the investigations were conducted.

\section{Experimental Protocol}

The resting metabolic rate (RMR) was measured $3 \mathrm{hr}$ after each subject had lunch. Each subject became accustomed to walking on a motor-driven treadmill until it became natural.
Afterward, the normal step frequency (step counts $\cdot \mathrm{min}^{-1}$ ) at which each subject feels comfortable walking (normal step frequency for each subject) was assessed by a researcher using a hand-tally counter at speeds of around 55,75 , and $95 \mathrm{~m} / \mathrm{min}$, which were generally used as low, normal, and high (brisk) walking speeds, respectively (Le Masurier and Tudor-Locke, 2003; Midorikawa et al., 2007). A low step frequency for each subject was calculated as $-15 \%$ of normal step frequency and a high step frequency as $+15 \%$. The $15 \%$ change in step frequency during walking on a treadmill was chosen based on the range of stride length that can be easily performed and is sufficient to significantly increase metabolic costs (Holt et al., 1991; Russell et al., 2010). Before starting the measurements, each subject practiced walking while matching it to the tempo (frequency) of an electronic metronome sound. Afterward, a facemask connected to the Douglas bag was worn by subjects with three accelerometers equipped on their waist; they then took a 5-minute rest while sitting on a chair mounted on the treadmill. The experimental procedure started at a $55 \mathrm{~m} / \mathrm{min}$ pace, and then was repeated at 75 and $95 \mathrm{~m} / \mathrm{min}$ in order. Three trials of normal, high, and low frequency were included at each walking speed (i.e., a total of nine tests were performed). The order of the nine tests was not randomized in order to assess them from lower to higher metabolic cost. A $15 \%$ decrease in stride length, i.e., increased step frequency, requires more energy expenditure compared with normal stride length (Russell et al., 2010) and longer steps at given walking speeds increase metabolic cost even more (Cavagna and Franzetti, 1986). The subject took a 5-min rest while seated between settings of step frequency and a 30-min rest between settings of walking speed. Also, we recorded the number of steps counted by three accelerometers during each trial and recorded all trials using a digital video recorder for precise counting later.

\section{Measurement of resting metabolic rate and metabolic equivalents}

Three hr after lunch, each subject sat relaxed for $30 \mathrm{~min}$ to reach stable oxygen consumption. RMR was then measured using a mask and Douglas bag for $20 \min (10 \min \times 2$ with a 1minute intermission). Energy expenditure during walking was measured using a mask and Douglas bag. Each subject walked for $6 \mathrm{~min}$ at $55 \mathrm{~m} / \mathrm{min}$ and $5 \mathrm{~min}$ at 75 or $95 \mathrm{~m} / \mathrm{min}$, and respiratory measurements were made during the last $3 \mathrm{~min}$ and $2 \mathrm{~min}$ at $55 \mathrm{~m} / \mathrm{min}$ and 75 or $95 \mathrm{~m} / \mathrm{min}$, respectively. $\mathrm{O}_{2}$ and $\mathrm{CO}_{2}$ concentrations of expired gas were measured using a gas analyzer (ARCO-1000A; Arco System, Kashiwa, Japan). Before each measurement, the gas analyzer was calibrated using room air and a certified gas. Expired gas volume was measured with a certified dry gas meter (SHINAGAWA DC-5, Tokyo, Japan). Energy expenditure (kcal) was calculated from $\mathrm{O}_{2}$ consumption and $\mathrm{CO}_{2}$ production using Weir's equation (Weir, 1949). The metabolic equivalents (METs) were determined by energy expenditure (kcal) obtained during walking divided by the measured RMR. 


\section{Equipment}

\section{Kenz Lifecorder EX}

The Kenz Lifecorder EX (LC; Suzuken Co., Ltd., Nagoya, Japan) is a uniaxial accelerometer, $70 \times 40 \times 25 \mathrm{~mm}$ in size and $30 \mathrm{~g}$ in mass. In this study, devices were attached on the side of the waist at the midline of the left thigh. This accelerometer samples at $32 \mathrm{~Hz}$ and assesses values ranging from 0.06 to $1.94 \mathrm{G}$. The LC uses only four thresholds from maximum amplitudes of vertical acceleration when determining the intensity levels. The signal is filtered by an analog bandpass filter and digitized. The maximum amplitude of the acceleration sensor and the step count generated by vertical movement determine the intensity levels. Ten intensity levels (0.5 and 1-9) are used to categorize intensities. The LC was initialized by setting the precise time and date and inputting the gender, age, height, and weight of each subject. After completing all trials, the data were downloaded using Physical Activity Analysis Software (Version 1.0, Suzuken Co., Ltd., Nagoya, Japan). The intensities obtained every $4 \mathrm{sec}$ were converted into METs using Kumahara's equation obtained during progressive walking and running on a treadmill (Kumahara et al., 2004). The relationship between METs and activity level was highly significant $\left(\mathrm{r}^{2}=0.929\right)$ :

METs $=0.043 \mathrm{x}^{2}+0.379 \mathrm{x}+1.361$ (Kumahara et al., 2004)

where $\mathrm{x}$ is the intensity level $(0.5,1-9$ intensities $)$.

\section{Actimarker}

The Actimarker (AM; Panasonic Electronic Works, Ltd., Osaka, Japan) is a triaxial accelerometer, $60 \times 35 \times 12 \mathrm{~mm}$ in size, and $30 \mathrm{~g}$ in mass. In the present study, devices were attached on either side of the waist at the midline of the left thigh. This device was released for sale in 2008 and has fieldproven reliability for estimating various activities (Yamada et al., 2009). The AM had user-friendly software providing activity intensity categories, daily energy expenditure, steps, and METs'hr. This device obtained three-dimensional accelerations with a sensitivity of $4 \mathrm{mG}$ and with a band-pass filter of 0.3 to $100 \mathrm{~Hz}$. The acceleration count was calculated as the average of the absolute values from acceleration in each direction for a given interval $(12 \mathrm{sec})$. The acceleration data were uploaded to a personal computer, and converted into METs by the following equation:

Physical Activity Energy Expenditure $(\mathrm{PAEE})(\mathrm{kcal} / \mathrm{min})=$ ax $\times[$ basal metabolic rate (BMR) $] / 1440+$ RMR

where $\mathrm{a}$ is a coefficient, and $\mathrm{x}$ is the output data from synthetic accelerations of 3 dimensions.

RMR is calculated by BMR $\times 1.2$

METs $=$ PAEE $/$ RMR.

The BMR was estimated according to the sixth Recommended Dietary Allowances for Japanese (Ministry of Welfare Japan, 1999). We also obtained the data of anteroposterior, mediolateral, and vertical accelerations from special software of the AM which was not available commercially.

\section{Active Style Pro}

The Active Style Pro (ASP; Omron Health Care Co., Ltd., Kyoto, Japan) is also a triaxial accelerometer, $80 \times 20 \times 50 \mathrm{~mm}$ in size, and $61 \mathrm{~g}$ in mass. The ASP was released for sale in Japan in 2008 and has proven reliability for estimating various activities (Oshima et al., 2010). In the present study, devices were attached on the right side of the waist. Anteroposterior, mediolateral, and vertical accelerations were obtained from the triaxial accelerometer during each activity with a sensitivity of $3 \mathrm{mG}$ and at a sampling rate of $32 \mathrm{~Hz}$. With a 12-bit analog to digital converter, the maximum scaling of the acceleration data was \pm 2048 counts. Acceleration data were then uploaded to a personal computer. The signals obtained from the triaxial accelerometer were processed in the following way. Each of the 3 signals from the triaxial accelerometer was passed through a high-pass filter with a cutoff frequency at $0.7 \mathrm{~Hz}$ to remove the gravitational acceleration component from the signal. We calculated the integral of the absolute value of the accelerometer output of each of the 3 axes using acceleration signals over a 10 -sec time interval. After the synthetic acceleration was filtered, it was categorized into either lifestyle or locomotive activity using a ratio of unfiltered to filtered synthetic acceleration. Synthetic accelerations of three dimensions were converted into METs by the follow equations: If Counts/min: $\leqq \mathrm{A}$, Sedentary METs is $\mathrm{b}+\mathrm{ax}$

If Counts/min: $>\mathrm{A}$ and Ratio: $\leqq \mathrm{B}$, lifestyle activity formula METs is $\mathrm{d}+\mathrm{cx}$

If Ratio: $>\mathrm{B}$, locomotive activity formula METs is $\mathrm{f}+\mathrm{ex}$ where $\mathrm{A}$ and $\mathrm{B}$ are thresholds, and a to $\mathrm{f}$ are coefficients. $\mathrm{x}$ is output data from synthetic accelerations of 3 dimensions.

\section{Statistics}

All values are presented as means \pm SD. Differences were considered to be statistically significant if the $p$ value was $<0.05$. Significant differences between men and women in the physical or gait characteristics were analyzed by an unpairedsample $t$-test. The percentage error was calculated as [(predicted value-observed value)/observed value] $\times 100$. Statistical comparisons of measured METs or the acceleration data obtained from the AM among step frequencies at each walking speed were performed by one-way analyses of variance (ANOVA) with repeated measures, and the Bonferroni procedure was used for post-hoc tests. One-way ANOVA with repeated measures was also used to compare differences between observed and predicted step counts or measured and predicted METs among accelerometers at each step frequency at a given walking speed; the Dunnett procedure was used for post-hoc tests. Two-way (step frequency and speed) ANOVA with repeated measures was used to determine how the factors affected the percentage error between measured and predicted METs in each accelerometer. When significant interactions were detected, simple main effect analysis was employed for each low, normal, or high step frequency to compare the effect of speed with the Bonferroni adjustment procedure. Multiple stepwise regression analysis 
Table 1 Physical characteristics of subjects

\begin{tabular}{lccc}
\hline & Men $(N=9)$ & Women $(N=9)$ & All subjects $(N=18)$ \\
\hline Age $(\mathrm{yr})$ & $31.0 \pm 4.9(24-37)$ & $28.1 \pm 5.9(23-41)$ & $29.6 \pm 5.5(23-41)$ \\
Height $(\mathrm{cm})$ & $171.6 \pm 2.2(167.6-173.9)$ & $161.5 \pm 5.9(151.4-167.9)^{*}$ & $166.5 \pm 6.8(151.4-173.9)$ \\
Body mass $(\mathrm{kg})$ & $71.2 \pm 6.5(62.3-78.2)$ & $52.7 \pm 6.2(45.3-59.2)^{*}$ & $71.2 \pm 6.5(45.3-78.2)$ \\
BMI $\left(\mathrm{kg} \cdot \mathrm{m}^{-2}\right)$ & $24.2 \pm 2.1(20.9-26.5)$ & $20.1 \pm 1.6(18.0-23.3)^{*}$ & $24.2 \pm 2.1(18.0-26.5)$ \\
$\mathrm{RMR}\left(\mathrm{kcal} \cdot \mathrm{min}^{-1}\right)$ & $1.1 \pm 0.3(0.77-1.59)$ & $0.9 \pm 0.1(0.76-1.01)$ & $1.1 \pm 0.3(0.76-1.59)$ \\
Step length $(\mathrm{cm})$ & & & \\
$55 \mathrm{~m} \cdot \mathrm{min}^{-1}$ & $57.2 \pm 5.2(50.9-66.3)$ & $58.2 \pm 5.9(51.4-67.9)$ & $57.2 \pm 5.2(50.9-67.9)$ \\
$75 \mathrm{~m} \cdot \mathrm{min}^{-1}$ & $67.7 \pm 4.2(60.0-74.3)$ & $69.0 \pm 4.9(59.5-74.0)$ & $67.7 \pm 4.2(59.5-74.3)$ \\
$95 \mathrm{~m} \cdot \mathrm{min}^{-1}$ & $79.4 \pm 4.9(70.4-85.6)$ & $78.3 \pm 6.1(64.6-82.6)$ & $79.4 \pm 4.9(64.6-85.6)$ \\
$\mathrm{Step} \mathrm{frequency}$ & & & \\
$55 \mathrm{~m} \cdot \mathrm{min}^{-1}$ & $96.8 \pm 8.5(83-108)$ & $95.3 \pm 9.5(81-107)$ & $96.8 \pm 8.5(81-108)$ \\
$75 \mathrm{~m} \cdot \mathrm{min}^{-1}$ & $111.1 \pm 7.1(101-125)$ & $109.2 \pm 8.3(101-126)$ & $111.1 \pm 7.1(101-126)$ \\
$95 \mathrm{~m} \cdot \mathrm{min}^{-1}$ & $120.1 \pm 7.7(111-135)$ & $122.1 \pm 10.6(110-147)$ & $120.1 \pm 7.7(110-147)$ \\
\hline
\end{tabular}

Abbreviations: BMI, body mass index; RMR, resting metabolic rate

Values are means \pm s.d. (range). $*$ indicates a significant difference from Men $(p<0.001)$.

Table 2 Accuracy of step-count functions among accelerometers

\begin{tabular}{|c|c|c|c|c|c|c|c|c|}
\hline \multirow{2}{*}{$\begin{array}{l}\text { walking } \\
\text { speed }\end{array}$} & \multirow{2}{*}{$\begin{array}{c}\text { Step } \\
\text { frequency }\end{array}$} & \multirow{2}{*}{$\begin{array}{l}\text { Visually counted } \\
\text { steps }\end{array}$} & \multicolumn{3}{|c|}{ Step detected } & \multicolumn{3}{|c|}{$p$ value } \\
\hline & & & $\mathrm{LC}$ & $\mathrm{AM}$ & ASP & $\mathrm{LC}$ & $\mathrm{AM}$ & ASP \\
\hline \multirow[t]{3}{*}{$55 \mathrm{~m} / \mathrm{min}$} & low & $492 \pm 45$ & $452 \pm 64$ & $410 \pm 97$ & $464 \pm 87$ & 0.171 & 0.001 & 0.414 \\
\hline & normal & $580 \pm 54$ & $534 \pm 86$ & $475 \pm 81$ & $576 \pm 64$ & 0.023 & $<0.001$ & 0.984 \\
\hline & high & $662 \pm 59$ & $616 \pm 72$ & $576 \pm 70$ & $650 \pm 58$ & 0.002 & $<0.001$ & 0.695 \\
\hline \multirow[t]{3}{*}{$75 \mathrm{~m} / \mathrm{min}$} & low & $475 \pm 31$ & $478 \pm 47$ & $466 \pm 48$ & $477 \pm 32$ & 0.968 & 0.693 & 0.997 \\
\hline & normal & $552 \pm 40$ & $538 \pm 49$ & $545 \pm 43$ & $551 \pm 40$ & 0.069 & 0.590 & 1.000 \\
\hline & high & $633 \pm 59$ & $595 \pm 113$ & $634 \pm 76$ & $633 \pm 60$ & 0.135 & 1.000 & 1.000 \\
\hline \multirow[t]{3}{*}{$95 \mathrm{~m} / \mathrm{min}$} & low & $522 \pm 40$ & $522 \pm 39$ & $521 \pm 40$ & $522 \pm 41$ & 0.999 & 0.969 & 0.994 \\
\hline & normal & $609 \pm 41$ & $606 \pm 40$ & $608 \pm 41$ & $609 \pm 41$ & 0.246 & 0.924 & 0.996 \\
\hline & high & $705 \pm 48$ & $692 \pm 56$ & $702 \pm 49$ & $701 \pm 47$ & 0.191 & 0.986 & 0.921 \\
\hline
\end{tabular}

Values are means \pm s.d. LC, Kenz Lifecorder; AM, Actimarker; ASP, Active Style Pro

was employed to determine which variables (step frequency, speed, step frequency x speed, sex, height, and body mass) contribute to the percentage error of estimating METs or step counts in each accelerometer. All statistical treatments were done using SPSS for Windows (version 16.0J; SPSS Inc., Chicago, IL, USA).

\section{Results}

The physical or gait characteristics of the subjects are shown in Table 1. Height, body mass, and BMI were significantly higher in men than in women $(p<0.001)$, whereas the other factors did not differ between genders.

\section{Accuracy of detecting step counts}

The LC significantly underestimated step counts at the normal or high step frequency at $55 \mathrm{~m} / \mathrm{min}$ (Table 2). The AM significantly underestimated step counts at all step frequencies at $55 \mathrm{~m} / \mathrm{min}$, whereas the ASP did not in any of the nine trials. In the percentage error between the measured and predicted step counts for each accelerometer, two-way ANOVA analysis demonstrated no significant interactions between step frequency and speed in all accelerometers (Fig. 1). Speed significantly contributed to the percentage error in all accelerometers whereas step frequency did not. A stepwise multiple regression analysis of predictors of the percentage error (including step frequency, speed, step frequency by speed, sex, height, and body mass) in the LC and AM revealed that speed was the only significant predictor $(\beta=0.32, p<$ 0.001 for the LC; $\beta=0.57, p<0.001$ for the AM), but no other factors were selected. The final models of the LC and AM accounted for $11 \%$ and $32 \%$ of the variance of the percentage error, respectively. In the case of the ASP, step frequency by speed was the only significant predictor $(\beta=0.21, p=0.007)$, and the final model accounted for $5 \%$ of the variance in percentage error.

\section{METs measured from Douglas bag method}

The METs measured at low, normal, and high step frequency were shown in Table 3. There were no differences in measured METs among the three step frequencies at $55 \mathrm{~m} / \mathrm{min}$. However, at 75 and $95 \mathrm{~m} / \mathrm{min} \quad(p=0.003$ and $p<0.001$, 
respectively), the measured METs at the low step frequency were significantly higher than those of the normal step frequency.

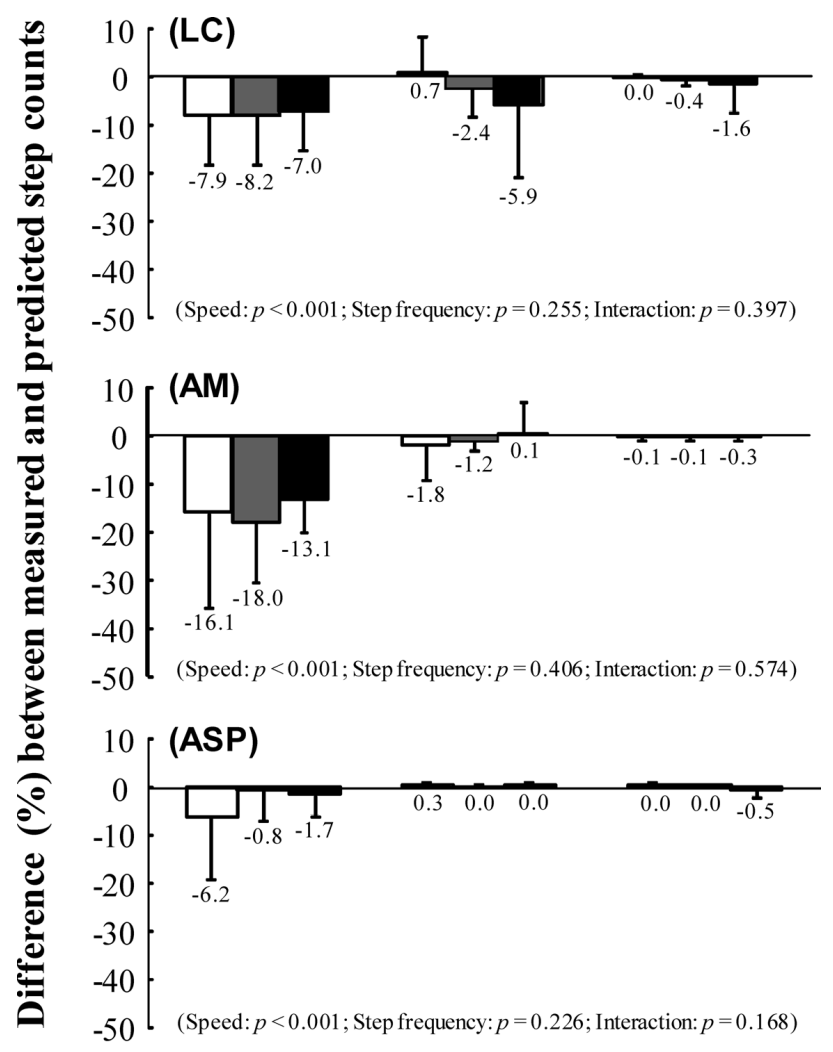

\section{$\frac{L N H}{55 \mathrm{~m} / \mathrm{min}} \quad \frac{\mathrm{L} \mathrm{N} \mathrm{H}}{75 \mathrm{~m} / \mathrm{min}} \quad \frac{\mathrm{L} H}{95 \mathrm{~m} / \mathrm{min}}$}

Fig. 1 Difference between measured (video records) and predicted step counts (LC, Kenz Lifecorder; AM, Actimarker; ASP, Active Style Pro) across conditions altered by three step frequencies (L, Low step frequency; N, Normal step frequency; H, High step frequency) at each walking speed $(55,75$, and $95 \mathrm{~m} / \mathrm{min})$. The numbers under the bars indicate the difference (\%) between measured and predicted step counts. The results of two-way ANOVA analysis were shown in parentheses for each accelerometer.

\section{Accuracy of predicted METs in accelerometers}

The LC significantly underestimated METs at the low and normal step frequencies at all walking speeds (Table 3). The AM significantly underestimated METs at the low step frequency at all walking speeds and also at the high step frequency of $95 \mathrm{~m} / \mathrm{min}$. The ASP significantly underestimated only at the low step frequency of $95 \mathrm{~m} / \mathrm{min}$. In the percentage error between the measured and predicted METs for each accelerometer, two-way ANOVA analysis demonstrated significant interaction between step frequency and speed only in the LC (Fig. 2). Simple main effect analysis in the LC showed no significant differences in the percentage errors among the three low step frequencies $(55 \mathrm{~m} / \mathrm{min}$ vs. $75 \mathrm{~m} / \mathrm{min}$ : $p=0.788 ; 75 \mathrm{~m} / \mathrm{min}$ vs. $95 \mathrm{~m} / \mathrm{min}: p=0.647 ; 55 \mathrm{~m} / \mathrm{min}$ vs. $95 \mathrm{~m} / \mathrm{min}: p=0.060$ ), the three normal step frequencies (all $p=1.000)$, or the three high step frequencies $(55 \mathrm{~m} / \mathrm{min}$ vs. $75 \mathrm{~m} / \mathrm{min}$ and $75 \mathrm{~m} / \mathrm{min}$ vs. $95 \mathrm{~m} / \mathrm{min}: p=1.000 ; 55 \mathrm{~m} / \mathrm{min}$ vs. $95 \mathrm{~m} / \mathrm{min}: p=0.612$ ). Step frequency significantly contributed to the percentage error in the AM and ASP, whereas speed did not. As shown in Table 4, a stepwise multiple regression analysis of predictors in the percentage error between the measured and predicted METs for each accelerometer revealed that step frequency was the strongest predictor in the LC. Speed and height were significantly associated, but step frequency by speed, sex, and body mass was not selected for the model. The final model accounted for 58\% of the model variation. The percentage error in the AM showed that height and sex significantly contributed to the percentage error, but step frequency, speed, and step frequency by speed were not selected. The final model accounted for $8.8 \%$ of the model variation. With ASP, the step frequency and speed significantly contributed to the percentage error, and the final model accounted for $10 \%$ of the model variation.

\section{Anteroposterior, mediolateral, and vertical accelerations}

Figure 3 shows the absolute data of anteroposterior, mediolateral, and vertical accelerations measured by the AM. Mediolateral acceleration at $55 \mathrm{~m} / \mathrm{min}$ was significantly higher at the high step frequency than at the normal step frequency. Mediolateral acceleration at $75 \mathrm{~m} / \mathrm{min}$ and $95 \mathrm{~m} / \mathrm{min}$ was

Table 3 Accuracy of predicted METs among accelerometers

\begin{tabular}{|c|c|c|c|c|c|c|c|c|}
\hline \multirow{2}{*}{$\begin{array}{c}\text { walking } \\
\text { speed }\end{array}$} & \multirow{2}{*}{$\begin{array}{c}\text { Step } \\
\text { frequency }\end{array}$} & \multirow{2}{*}{$\begin{array}{c}\text { Observed METs } \\
\text { steps }\end{array}$} & \multicolumn{3}{|c|}{ Predicted METs } & \multicolumn{3}{|c|}{$p$ value } \\
\hline & & & $\mathrm{LC}$ & $\mathrm{AM}$ & ASP & $\mathrm{LC}$ & $\mathrm{AM}$ & ASP \\
\hline \multirow[t]{3}{*}{$55 \mathrm{~m} / \mathrm{min}$} & low & $3.2 \pm 0.9$ & $2.2 \pm 0.1$ & $2.8 \pm 0.3$ & $3.0 \pm 0.3$ & $<0.001$ & 0.035 & 0.643 \\
\hline & normal & $2.8 \pm 0.4$ & $2.5 \pm 0.3$ & $2.7 \pm 0.2$ & $3.0 \pm 0.2$ & 0.009 & 0.411 & 0.096 \\
\hline & high & $3.0 \pm 0.7$ & $2.9 \pm 0.4$ & $2.8 \pm 0.3$ & $3.2 \pm 0.3$ & 0.866 & 0.250 & 0.616 \\
\hline \multirow[t]{3}{*}{$75 \mathrm{~m} / \mathrm{min}$} & low & $4.1 \pm 0.7$ & $2.6 \pm 0.3$ & $3.5 \pm 0.3$ & $3.9 \pm 0.3$ & $<0.001$ & 0.002 & 0.567 \\
\hline & normal & $3.6 \pm 0.7$ & $3.1 \pm 0.4$ & $3.3 \pm 0.3$ & $3.9 \pm 0.4$ & 0.004 & 0.151 & 0.273 \\
\hline & high & $3.8 \pm 0.9$ & $4.0 \pm 1.0$ & $3.4 \pm 0.5$ & $4.0 \pm 0.7$ & 0.714 & 0.178 & 0.616 \\
\hline \multirow[t]{3}{*}{$95 \mathrm{~m} / \mathrm{min}$} & low & $6.0 \pm 1.0$ & $3.3 \pm 0.4$ & $4.7 \pm 0.3$ & $5.2 \pm 0.4$ & $<0.001$ & $<0.001$ & 0.001 \\
\hline & normal & $4.5 \pm 0.6$ & $4.0 \pm 0.8$ & $4.2 \pm 0.3$ & $4.9 \pm 0.4$ & 0.010 & 0.093 & 0.141 \\
\hline & high & $4.7 \pm 0.8$ & $5.1 \pm 0.6$ & $4.2 \pm 0.5$ & $5.1 \pm 0.6$ & 0.074 & 0.045 & 0.168 \\
\hline
\end{tabular}

Values are means \pm s.d. METs, Metabolic equivalents; LC, Kenz Lifecorder; AM, Actimarker; ASP, Active Style Pro 


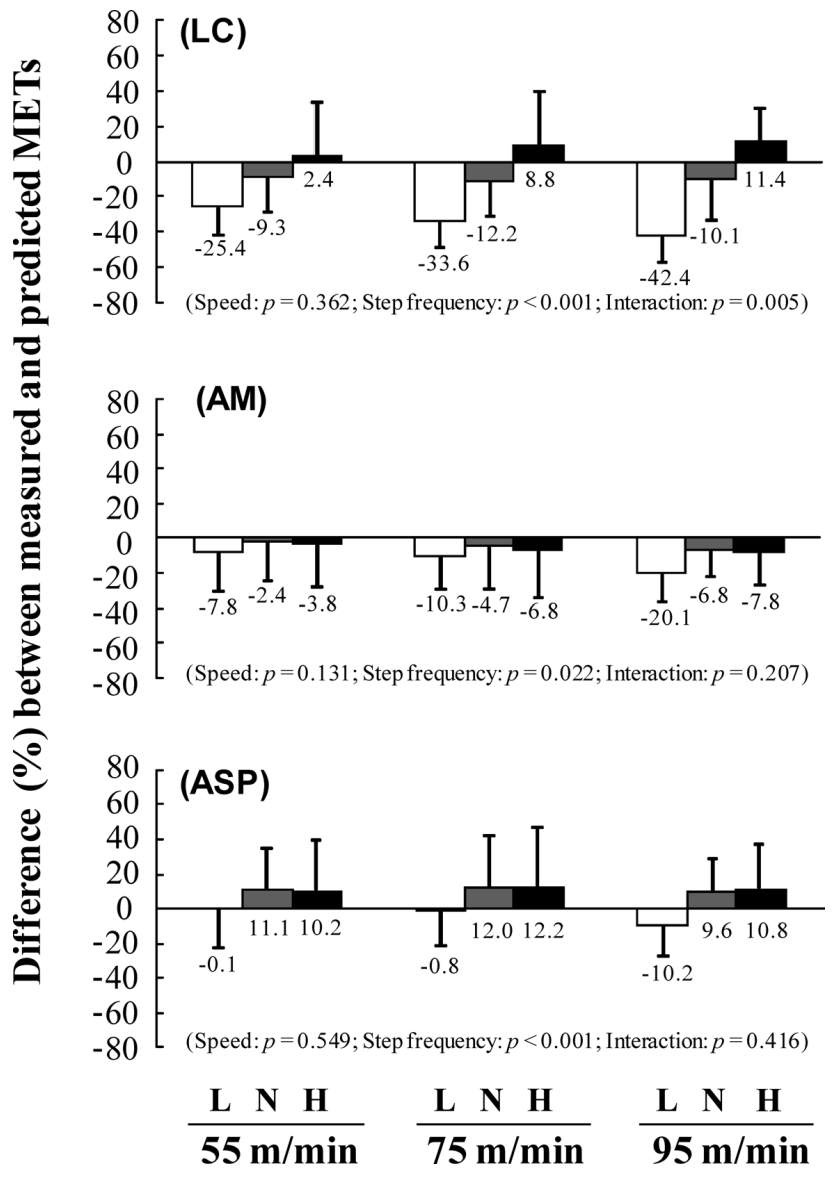

Fig. 2 Difference between measured (Douglas bag) and predicted METs (LC, Kenz Lifecorder; AM, Actimarker; ASP, Active Style Pro) across conditions altered by three step frequencies (L, Low step frequency; $\mathrm{N}$, Normal step frequency; H, High step frequency) at each walking speed $(55,75$, and $95 \mathrm{~m} / \mathrm{min})$. The numbers under the bars indicate the difference $(\%)$ between measured and predicted METs. The results of two-way ANOVA analysis were shown in parentheses for each accelerometer. significantly higher at the low step frequency than at the normal frequency. Vertical acceleration was significantly higher at the high step frequency at $55 \mathrm{~m} / \mathrm{min}$ or at the low step frequency at $95 \mathrm{~m} / \mathrm{min}$ than at the normal frequency. Anteroposterior acceleration was significantly higher at the low step frequency at all walking speeds when compared to normal frequency. The total value of three accelerations was significantly higher at the high step frequency at $55 \mathrm{~m} / \mathrm{min}$ than at the normal frequency and also higher at the low step

Table 4 Multiple regression analysis of predictors in the percentage error between the measured and predicted METs in each accelerometer

\begin{tabular}{|c|c|c|c|c|c|c|}
\hline \multirow{2}{*}{ Predictors } & \multirow{2}{*}{$\mathrm{R}$} & \multirow{2}{*}{$\mathrm{R}^{2}$} & \multicolumn{2}{|c|}{$\begin{array}{l}\text { Unstandardized } \\
\text { coefficients }\end{array}$} & \multirow{2}{*}{$\beta$} & \multirow{2}{*}{$p$-value } \\
\hline & & & B & $\begin{array}{l}\text { Standard } \\
\text { error }\end{array}$ & & \\
\hline \multicolumn{7}{|l|}{$\mathrm{LC}$} \\
\hline Step frequency & & & 1.262 & 0.089 & 0.869 & $<0.001$ \\
\hline Speed & & & -0.857 & 0.102 & -0.514 & $<0.001$ \\
\hline Height & & & -0.550 & 0.214 & -0.133 & $<0.001$ \\
\hline Total & 0.764 & 0.584 & & & & $<0.001$ \\
\hline \multicolumn{7}{|l|}{$\mathrm{AM}$} \\
\hline Height & & & -1.409 & 0.370 & -0.452 & $<0.001$ \\
\hline Sex & & & -11.832 & 4.880 & -0.288 & 0.016 \\
\hline Total & 0.296 & 0.088 & & & & $<0.001$ \\
\hline \multicolumn{7}{|l|}{ ASP } \\
\hline Step frequency & & & 0.487 & 0.116 & 0.374 & $<0.001$ \\
\hline Speed & & & -0.396 & 0.134 & -0.265 & 0.004 \\
\hline Total & 0.322 & 0.103 & & & & $<0.001$ \\
\hline
\end{tabular}

Abbreviations: METs, Metabolic equivalents; R, Multiple correlation coefficient; $\mathrm{R}^{2}$, Multiple coefficient of determination; $\beta$, Standardized partial regression coefficient; LC, Kenz Lifecorder; AM, Actimarker; ASP, Active Style Pro.

Confounding factors of step frequency, speed, step frequency x speed, sex, height, and body mass were used in the analyses of each accelerometer.
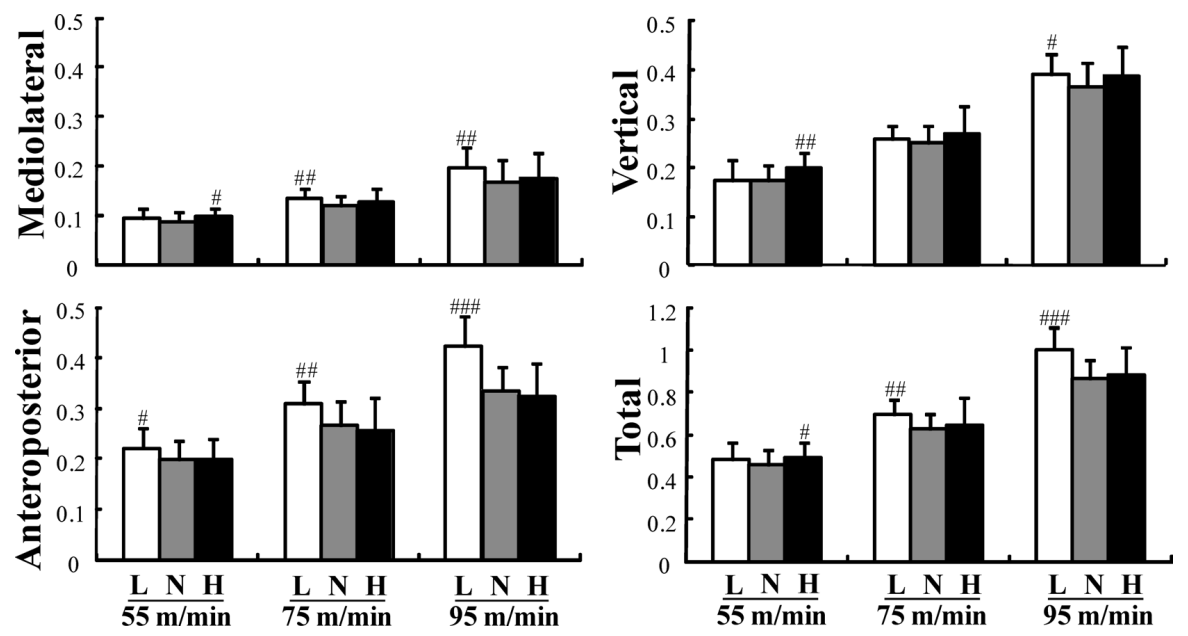

Fig. 3 Absolute accelerations in the mediolateral, vertical, anteroposterior, and total directions measured by the Actimarker across conditions altered by three step frequencies (L, Low step frequency; N, Normal step frequency; H, High step frequency) at each walking speed $(55 \mathrm{~m} / \mathrm{min}, 75 \mathrm{~m} / \mathrm{min}, 95 \mathrm{~m} / \mathrm{min})$. Values significantly different from $\mathrm{N}$ (Normal step frequency) are indicated by ${ }^{\#} p<0.05,{ }^{\#} p<0.01$, \#\#\# $p<0.001$. 
frequency at 75 and $95 \mathrm{~m} / \mathrm{min}$.

\section{Discussion}

This is the first study to investigate whether changes in step frequency have independent effects on the validity of stepcount functions and predicted energy cost assessed by accelerometers. The LC underestimated the step counts at normal or high step frequency at low walking speed. The AM also underestimated them at all step frequencies at low walking speed, whereas the ASP did not across any of the trials. The degree of the percentage error of the step counts in all accelerometers was affected by speed, but not by step frequency. The LC underestimated METs at the low or normal step frequency at all walking speeds, whereas overall underestimation was less across trials in the AM and ASP.

The present study clearly demonstrated that LC, AM, and ASP have very accurate step-count functions at normal walking speed with normal step frequency at which each subject feels comfortable walking. Schneider et al. (2003) demonstrated that LC had more accurate step-count functions compared with 7 other pedometers during a $400-\mathrm{m}$ track walk at self-selected speeds for adults, and its error in detecting actual steps taken was within $\pm 3 \%$. Actually, Cao et al. (2010) reported no significant differences between LC and AM in daily walking step counts for adults. The error of $-2.4 \%$ (LC), $-1.2 \%$ (AM), and $0.0 \%$ (ASP) at normal walking speed with normal step frequency in the present study meet the Japanese Industrial Standard set by the Ministry of Industry and Trading criteria indicating that error should be within 3\% (3 steps of 100) (Hatano, 1993). Therefore, it is considered that LC, AM, and ASP are among the pedometers which are very accurate and sufficiently reliable to administer to large groups.

At low walking speed, the LC underestimated the step counts at the normal and high frequency while the AM underestimated them at all step frequencies. In contrast, the ASP had accurate step-count functions across all trials. The present results correspond with those of other studies using electronic pedometers that underestimated step counts at walking speeds slower than about $55 \mathrm{~m} / \mathrm{min}$ (Crouter et al., 2003; Le Masurier and Tudor-Locke, 2003; Le Masurier et al., 2004). Thus, the impact on the accelerometers (sensitivity) during slow walking for the LC and AM might be too weak to detect a "threshold" of capturing a step, whereas ASP had better reliability in detecting step counts even with slow walking. It was difficult to determine why the ASP had better accuracy than the AM in detecting step counts; however, the difference in the filtering system between the AM and the ASP might explain this. Even so, speed significantly contributed to the degree of the percentage error in all three accelerometers. Therefore, we suggest that accelerometers should be carefully used when assessing daily step counts, especially for persons who walk slowly.

The present study revealed that the LC underestimated METs at the low and normal step frequency at all walking speeds, and gross underestimation was found especially at low step frequency of high walking speeds. One possible explanation for the LC error is its own proprietary dataanalyzing process, in which intensity levels are categorized using both the step counts and the maximum amplitude of vertical acceleration every four seconds. In this study, although higher energy cost was demanded at a low step frequency especially at a higher walking speed, the number of step counts by the LC would have conversely decreased due to the greater step length (lower step frequency). Therefore, a decrease in step counts at the low step frequency might cause the LC underestimation. The possibility that step frequency could strongly affect the validation of the LC was supported by the following results: Step frequency was the strongest predictor ( $\beta=0.87)$ and speed was the second strongest $(\beta=-0.51)$ of the error between the measured and predicted METs in the multiple regression analysis. Therefore, changes in step frequency would individually and markedly affect the accuracy of the LC.

Another possible reason for the LC underestimation may be that in the data-analyzing process, it uses only four thresholds from maximum amplitudes of vertical acceleration when determining the intensity levels (i.e., noncontinuous variables). For example, if the maximum amplitudes of vertical acceleration during walking altered by both low and normal step frequency at a fixed walking speed are between $0.15-0.76$ $\mathrm{G}$, the difference in intensity levels between the low and normal step frequency will be determined by the difference in the step counts. However, as mentioned above, the number of step counts by the LC would have been decreased due to the low step frequency, despite the higher energy cost. This might be one explanation for the $\mathrm{LC}$ error.

Compared with the results of the LC, the AM and ASP showed less error in measuring METs across trials. Multiple regression analysis indicated that step frequency did not affect AM accuracy. Although ASP accuracy was affected by step frequency, it only explains $10 \%$ of the error. Better validity of the AM and ASP compared with the LC might be partly due to the higher capability of the triaxial accelerometers in assessing multiple-directional accelerations as continuous variables. In the earlier studies, anteroposterior or vertical acceleration contributed to a highly accurate estimation of physical activity under normal walking conditions in which step frequency was concurrently changed with an increment in speed (Bouten et al., 1994; Kumahara et al., 2004). However, in our experimental protocol (i.e., various step frequencies altered at a fixed walking speed), the difference in vertical acceleration between the low and normal step frequency was much less than the difference between the low and normal step frequencies in the METs measured by the Douglas bag method. Moreover, the major acceleration component at a low step frequency was in the anteroposterior direction, but in the vertical direction at a high step frequency. Based on our results, we suggest that the AM and ASP assure more accuracy than the LC for estimating intensity or energy costs under various walking conditions. 
In the present study, the degree of the percentage error of METs was affected by step frequency both in the AM and ASP. Significant underestimation was found in AM at all low step frequencies of all walking speeds, but in ASP only at low step frequency of high walking speed. As shown in the raw data of the three accelerations, the total values using the output of the three accelerations at the high walking speed was around $16 \%$ higher at the low step frequency than at the normal step frequency. However, the difference in the measured METs from the Douglas bag at the high walking speed was around $25 \%$ higher at the low step frequency than at the normal step frequency. The discrepancy of $16 \%$ and $25 \%$ might therefore result in higher error at the low step frequency of high walking speed in the AM and ASP. Furthermore, the present study showed that METs estimated by the AM tended to be entirely underestimated across trials compared with METs estimated by the ASP (Fig. 2). Because the minimum amplitude of the acceleration sensor was similar between the AM $(4 \mathrm{mG})$ and the ASP $(3 \mathrm{mG})$, the sensitivity of the minimum amplitude of the acceleration sensor did not affect the error of the AM. Therefore, we consider that AM accuracy may be improved by using more suitable equations to precisely measure energy costs altered by the various walking patterns.

The present study has the following limitations. First, our results using young healthy subjects might not be readily generalized to children or older adults due to different characteristics such as the length and mass of legs and body movement. In addition, the elderly have been known to walk and step so slowly that their walking movements are greater in mediolateral directions (Dean et al., 2007). Therefore, further research is needed to evaluate the accuracy of an accelerometer for other aged subjects under the same conditions and to calibrate for more accurate estimations. Second, we cannot exclude differences between walking on a treadmill indoors and freely walking outdoors. However, in general, the energy costs of treadmill and over-ground walking on a firm surface are similar (Hall et al., 2004). Hence, we thought that using the treadmill in our experimental protocol was adequate to obtain more precise and reliable data as a basic study.

In conclusion, these results suggest that accelerometers can cause errors in step-count functions at a low walking speed. Furthermore, in the measurement of energy costs, LC may cause great errors especially for the group with various step frequency and speed, whereas AM and ASP, which are tri-axial accelerometers, cause fewer errors but the degree of the percentage error is affected by step frequency.

Acknowledgements The authors thank the study subjects of the National Institute of Health and Nutrition for their cooperation. We especially thank Y Hikihara, K Ohkawara, Y Azuma, L Miyake, and $\mathrm{H}$ Kogure for their invaluable assistance in the present experiment. This work was performed as part of the Health and Labor Sciences Research Grants (Comprehensive Research on Cardiovascular and Life-style Related Diseases) from the Ministry of Health, Labor and
Welfare of Japan (to S Tanaka), and was supported, in part, by the Suzuken Memorial Foundation (to Ishikawa-Takata K).

\section{References}

Bassett DR Jr, Mahar MT, Rowe DA, Morrow JR Jr (2008) Walking and measurement. Med Sci Sports Exerc 7 (Suppl): S529-536

Bouten CV, Westerterp KR, Verduin M, Janssen JD (1994) Assessment of energy expenditure for physical activity using a triaxial accelerometer. Med Sci Sports Exerc 26: 1516-1523

Bravata DM, Smith-Spangler C, Sundaram V, Gienger AL, Lin N, Lewis R, Stave CD, Olkin I, Sirard JR (2007) Using pedometers to increase physical activity and improve health: a systematic review. JAMA 298: 2296-2304

Cao ZB, Miyatake N, Higuchi M, Miyachi M, Ishikawa-Takata $\mathrm{K}$, Tabata I (2010) Predicting $\mathrm{VO}_{2}$ max with objectively measured physical activity in Japanese women. Med Sci Sports Exerc 42: 179-186

Cavagna GA, Franzetti P (1986) The determinants of the step frequency in walking in humans. J Physiol 373: 235-242

Crouter SE, Schneider PL, Karabulut M, Bassett DR Jr (2003) Validity of 10 electronic pedometers for measuring steps, distance, and energy cost. Med Sci Sports Exerc 35: 1455-1460

Dean JC, Alexander NB, Kuo D (2007) The effect of lateral stabilization on walking in young and old adults. IEEE Trans Biomed Eng 54: 1919-1926

Dillman CJ (1975) Kinematic analyses of running. Exerc Sport Sci Rev 3: 193-218

Hakim AA, Curb JD, Petrovitch H, Rodriguez BL, Yano K, Ross GW, White LR, Abbott RD (1999) Effects of walking on coronary heart disease in elderly men: the Honolulu Heart Program. Circulation 100: 9-13

Hall C, Figueroa A, Fernhall B, Kanaley JA (2004) Energy expenditure of walking and running: comparison with prediction equations. Med Sci Sports Exerc 36: 2128-2134

Hatano Y (1993) Use of the pedometer for promoting daily walking exercise. ICHPER J 29: 4-8

Holt KG, Hamill J, Andres RO (1991) Predicting the minimal energy costs of human walking. Med Sci Sports Exerc 23: 491-498

Kumahara H, Schutz Y, Ayabe M, Yoshioka M, Yoshitake Y, Shindo M, Ishii K, Tanaka H (2004) The use of uniaxial accelerometry for the assessment of physical-activity-related energy expenditure: a validation study against whole-body indirect calorimetry. Br J Nutr 91: 235-243

Lee IM, Buchner DM (2008) The importance of walking to public health. Med Sci Sports Exerc 40(Suppl): S512-18

Le Masurier GC, Tudor-Locke C (2003) Comparison of pedometer and accelerometer accuracy under controlled conditions. Med Sci Sports Exerc 35: 867-871

Le Masurier GC, Lee SM, Tudor-Locke C (2004) Motion sensor accuracy under controlled and free-living conditions. 
Med Sci Sports Exerc 36: 905-910

McClain JJ, Craig CL, Sisson SB, Tudor-Locke C (2007a) Comparison of Lifecorder EX and ActiGraph accelerometers under free-living conditions. Appl Physiol Nutr Metab 32: 753-761

McClain JJ, Sisson SB, Washington TL, Craig CL, TudorLocke C (2007b) Comparison of Kenz Lifecorder EX and ActiGraph accelerometers in 10-yr-old children. Med Sci Sports Exerc 39: 630-638

Midorikawa T, Tanaka S, Kaneko K, Koizumi K, IshikawaTakata K, Futami J, Tabata I (2007) Evaluation of lowintensity physical activity by triaxial accelerometry. Obesity 15: 3031-3038

Ministry of Welfare Japan (1999) Recommended Dietary Allowances for the Japanese 6th ed. Ministry of Welfare, Japan, 36 [In Japanese]

Mitsui T, Shimaoka K, Tsuzuku S, Kajioka T, Sakakibara H (2008) Pedometer-determined physical activity and indicators of health in Japanese adults. J Physiol Anthropol 27: 179-184

Oshima Y, Kawaguchi K, Tanaka S, Ohkawara K, Hikihara Y, Ishikawa-Takata K, Tabata I (2010) Classifying household and locomotive activities using a triaxial accelerometer. Gait Posture 31: 370-374

Plasqui G, Westerterp KR (2007) Physical activity assessment with accelerometers: an evaluation against doubly labeled water. Obesity 15: 2371-2379

Rowlands AV, Stone MR, Eston RG (2007) Influence of speed and step frequency during walking and running on motion sensor output. Med Sci Sports Exerc 39: 716-727

Russell EM, Braun B, Hamill J (2010) Does stride length influence metabolic cost and biomechanical risk factors for knee osteoarthritis in obese women? Clin Biomech 25: 438-443

Schneider PL, Crouter SE, Lukajic O, Bassett DR (2003) Accuracy and reliability of 10 pedometers for measuring steps over a 400-m walk. Med Sci Sports Exerc 35: 1779-1784

Schneider PL, Crouter SE, Bassett DR (2004) Pedometer measures of free-living physical activity: comparison of 13 models. Med Sci Sports Exerc 36: 331-335

Weir, JBdV (1949) New methods for calculating metabolic rate with special reference to protein metabolism. J Physiol 109: $1-9$

Yamada Y, Yokoyama K, Noriyasu R, Osaki T, Adachi T, Itoi A, Naito Y, Morimoto T, Kimura M, Oda S (2009) Lightintensity activities are important for estimating physical activity energy expenditure using uniaxial and triaxial accelerometers. Eur J Appl Physiol 105: 141-152

Received: August 11, 2010

Accepted: February 15, 2011

Correspondence to: Jonghoon Park, Health Promotion and Exercise Program, National Institute of Health and Nutrition, 1-23-1 Toyama, Shinjuku-ku, Tokyo 162-8636, Japan

Phone: +81-3-3203-8061

Fax: +81-3-3203-1731

e-mail: jonghoon@nih.go.jp 